\title{
Subwavelength resolution with a negative-index metamaterial superlens
}

Koray Aydin, Irfan Bulu, and Ekmel Ozbay

Citation: Appl. Phys. Lett. 90, 254102 (2007); doi: 10.1063/1.2750393

View online: http://dx.doi.org/10.1063/1.2750393

View Table of Contents: http://aip.scitation.org/toc/apl/90/25

Published by the American Institute of Physics

\section{Articles you may be interested in}

Imaging properties of a metamaterial superlens

Applied Physics Letters 82, 161 (2003); 10.1063/1.1536712

Electric-field-coupled resonators for negative permittivity metamaterials

Applied Physics Letters 88, 041109 (2006); 10.1063/1.2166681

Observation of negative refraction and negative phase velocity in left-handed metamaterials

Applied Physics Letters 86, 124102 (2005); 10.1063/1.1888051

Rapid growth of evanescent wave by a silver superlens

Applied Physics Letters 83, 5184 (2003); 10.1063/1.1636250

Microwave transmission through a two-dimensional, isotropic, left-handed metamaterial Applied Physics Letters 78, 489 (2001); 10.1063/1.1343489

Limitations on subdiffraction imaging with a negative refractive index slab

Applied Physics Letters 82, 1506 (2003); 10.1063/1.1554779

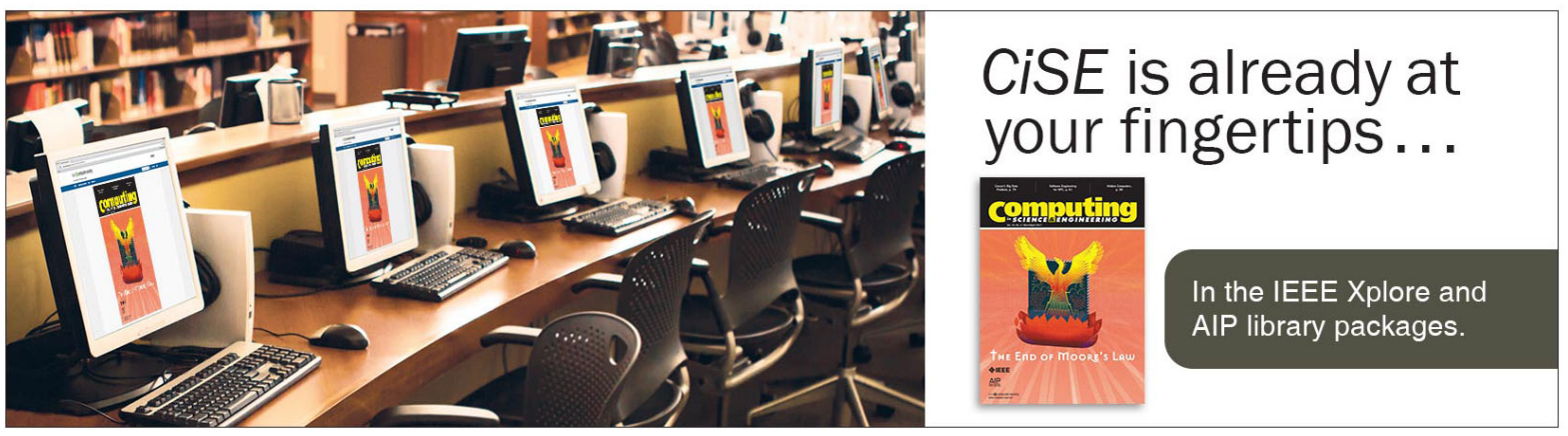




\title{
Subwavelength resolution with a negative-index metamaterial superlens
}

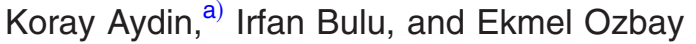 \\ Nanotechnology Research Center-NANOTAM, Bilkent University, Bilkent, 06800 Ankara, Turkey; \\ Department of Physics, Bilkent University, Bilkent, 06800 Ankara, Turkey; and Department of Electrical \\ and Electronics Engineering, Bilkent University, Bilkent, 06800 Ankara, Turkey
}

(Received 8 March 2007; accepted 26 May 2007; published online 19 June 2007)

\begin{abstract}
Negative-index metamaterials are candidates for imaging objects with sizes smaller than a half-wavelength. The authors report an impedance-matched, low loss negative-index metamaterial superlens that is capable of resolving subwavelength features of a point source with a $0.13 \lambda$ resolution, which is the highest resolution achieved by a negative-index metamaterial. By separating two point sources with a distance of $\lambda / 8$, they were able to detect two distinct peaks on the image plane. They also showed that the metamaterial based structure has a flat lens behavior. (c) 2007 American Institute of Physics. [DOI: 10.1063/1.2750393]
\end{abstract}

Refraction is a basic phenomenon that is widely used in electromagnetism and optics, and forms the basis of imaging process and lenses. Ubiquitous materials have positive refractive indices; however, it is possible to obtain negative values of refractive index by making use of the concept of metamaterials and photonic crystals. ${ }^{1,2}$ The possibility of bringing the refractive index into the domain of negative values was first discussed by Veselago ${ }^{3}$ in which the first steps to realize these exciting materials were taken four decades later. ${ }^{4}$ Inspired by the intriguing electromagnetic (EM) properties arising from negative-index metamaterials (NIMs), these types of artificially constructed structures received burgeoning interest from the scientific community. ${ }^{5-11}$

A perfect lens is one of the most important applications of materials with a negative refractive index. The term, perfect lens, was coined by Pendry owing to the ability of such lenses to reconstruct a perfect image by recovering the evanescent components of EM waves. ${ }^{12}$ In conventional optics, the lenses are constructed from positive-index materials and require curved surfaces to bring EM waves into focus. Positive-index lenses act only on the phase of the radiation, thus their resolution is limited to the half-wavelength. The finer details of the image are carried by high- $k$ components, the so called evanescent waves, and quickly decay before reaching the image plane. Therefore, the contribution of evanescent components to the resolution of the image is absent in conventional lenses. However, Pendry conceived that it is possible to enhance evanescent waves by using a negative refractive index medium. Photonic crystals $^{13}$ and metamaterials $^{6-10,14,15}$ were experimentally demonstrated to achieve diffraction-free imaging, in which further theoretical studies ${ }^{16-18}$ supported these results. In this letter, we demonstrate an impedance-matched, low loss negative-index metamaterial superlens that is capable of resolving subwavelength features with a record-level $0.13 \lambda$ resolution, which is the highest resolution achieved by a negative-index metamaterial. In our study, we employed a two-dimensional (2D) metamaterial based on split ring resonator (SRR) and wire geometry to achieve subwavelength resolution.

The NIM under investigation is a slab of 2D SRR-wire arrays deposited on FR4 printed circuit boards [Fig. 1(a)], with the parameters provided in Ref. 10. The NIM slab has

\footnotetext{
${ }^{a)}$ Electronic mail: aydin@fen.bilkent.edu.tr
}

$40 \times 20 \times 3$ layers along the $x, y$, and $z$ directions with equal lattice constants in all directions, $a_{x}=a_{y}=a_{z}=9.3 \mathrm{~mm}$. The NIM slab has a thickness of $2.79 \mathrm{~cm}(\sim \lambda / 3)$ and a length of $38 \mathrm{~cm}(4.8 \lambda)$. Transmission and reflection measurements are performed to characterize 2D NIM, in which the results are plotted in Fig. 1(b). A well-defined transmission peak is observed between 3.65 and $4.00 \mathrm{GHz}$, where the effective permeability and effective permittivity of NIM are simultaneously negative. ${ }^{10} \mathrm{~A}$ sharp dip in the reflection spectrum is observed at $3.78 \mathrm{GHz}$. The reflection is very low, $-37 \mathrm{~dB}$, meaning that the incident EM waves nearly do not face any reflection at the NIM surface.

The effective parameters of the NIM are retrieved by using the calculated amplitudes and phases of transmission and reflection by following the method in Ref. 19 The ambiguity in the determination of the correct branch is resolved by use of an analytic continuation procedure. ${ }^{19}$ Dielectric permittivity $\varepsilon=\varepsilon^{\prime}+i \varepsilon^{\prime \prime}$ and magnetic permeability $\mu=\mu^{\prime}$ $+i \mu^{\prime \prime}$ are used to describe the response of materials to the incident electromagnetic field, where $\varepsilon^{\prime}$ and $\mu^{\prime}$ are the real parts, and $\varepsilon^{\prime \prime}$ and $\mu^{\prime \prime}$ are the imaginary parts of the corresponding effective parameters. Figure 2(a) depicts the real parts of $\varepsilon$ (blue line) and $\mu$ (red line), and simulated reflection spectrum (black line). $\varepsilon^{\prime}$ and $\mu^{\prime}$ possess negative values between 3.63 and $3.93 \mathrm{GHz}$. The minimum reflection in the simulations occurs at $3.74 \mathrm{GHz}$, where $\varepsilon^{\prime}=\mu^{\prime}=-1.8$ (dashed orange line). The real and imaginary parts of the index of refraction $n^{\prime}$ (blue line) and $n^{\prime \prime}$ (green line) and the real part of impedance $Z^{\prime}$ (red line) are plotted in Fig. 2(b). The im-
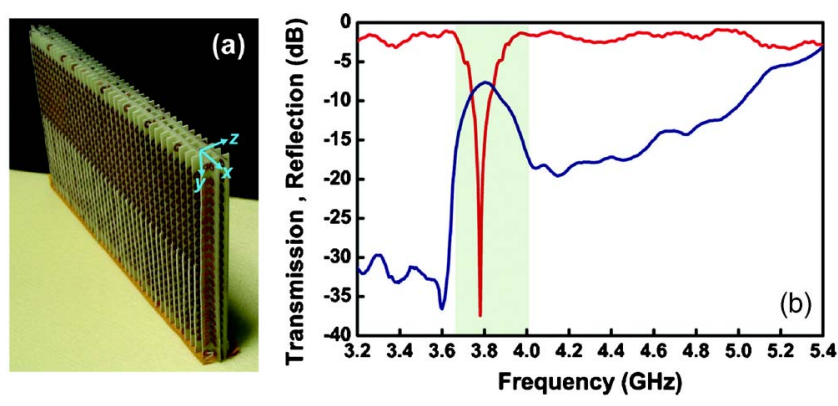

FIG. 1. (Color online) (a) Photograph of NIM slab with three unit cells along the $z$ direction. (b) Measured transmission (blue) and reflection (red) spectra for a NIM slab. 

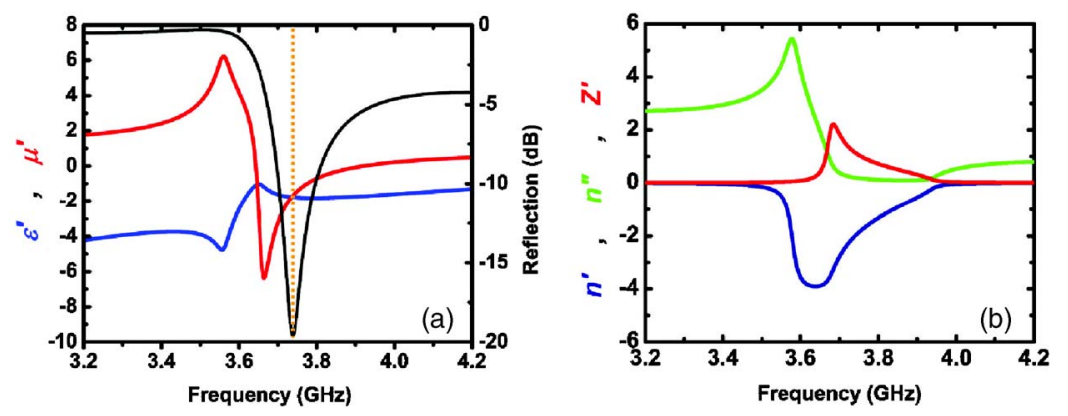

FIG. 2. (Color online) (a) Real parts of retrieved effective permittivity (blue) and permeability (red), and reflection spectrum (black). (b) Real parts of a retrieved refractive index (blue), impedance (red), and imaginary part of the refractive index (green). pedance is defined as $Z=\sqrt{\mu^{\prime} / \varepsilon^{\prime}}$; therefore impedance matching is obtained when $\varepsilon^{\prime}=\mu^{\prime}$. Expectedly, the impedance of NIM is matched to that of free space at $3.74 \mathrm{GHz}$, where $Z^{\prime}=1$.

The imaging measurements presented here are performed at $3.78 \mathrm{GHz}$, where the reflection is considerably low and the losses due to reflection are negligible. The NIM has a refractive index of $n_{\text {eff }}=-2.07 \pm 0.22$ at $3.78 \mathrm{GHz}$, which is measured by using a wedge-shaped 2D NIM. ${ }^{10}$ The refractive index obtained from the retrieval procedure is $n_{\mathrm{eff}}=$ -1.81 . Figure of merit is defined as the ratio of $n^{\prime}$ to $n^{\prime \prime}$ and used to characterize the performance of NIMs. ${ }^{20-22}$ In our simulations, we found $n^{\prime}=-1.81$ and $n^{\prime \prime}=-0.15$ at $3.74 \mathrm{GHz}$. Therefore, the figure of merit is 12 , the highest value ever reported. The NIM structure has low absorption losses, and therefore can be used to achieve subwavelength resolution.

In the imaging experiments, we employed monopole antennae to imitate the point source. The exposed center conductor acts as the transmitter and receiver and has a length of $4 \mathrm{~cm}(\sim \lambda / 2)$. Firstly, we measured the beam profile in free space that is plotted in Fig. 3(a) with a red dashed line. The full width at half maximum of the beam is $8.2 \mathrm{~cm}(1.03 \lambda)$. Then, we inserted NIM superlens and measured the spot size of the beam as $0.13 \lambda$, which is well below the diffraction limit. The source is located $d_{s}=1.2 \mathrm{~cm}$ away from first boundary and the image forms $d_{i}=0.8 \mathrm{~cm}$ away from second boundary of the superlens. The intensity of the electric field at the image plane is scanned by the receiver monopole antenna with $\Delta x=2 \mathrm{~mm}$ steps. The field intensity is normalized with respect to the maximum intensity in figure.

However, this focusing behavior could have been due to a channeling effect. The SRR-wire boards are separated with $9.3 \mathrm{~mm}$ and the field may propagate on these channels. To ensure that the subwavelength imaging is due to the effective response of NIM and not that of individual channels, we moved the point source along the source plane to check the flat lens behavior. ${ }^{23}$ The resulting intensity distributions are plotted in Fig. 3(b) for different source locations, namely, $x$ $=0 \mathrm{~cm}$ (black line), $0.5 \mathrm{~cm}$ (red line), and $-1.3 \mathrm{~cm}$ (gray line). In all the cases, the images were formed exactly at the same $x$ distance with the source. It is noteworthy that the distances are not the multiples of the lattice constant, i.e., the sources are not located on the axes of SRR-wire boards.

We used two point sources separated by distances smaller than a wavelength to obtain subwavelength resolution. The sources are driven by two independent signal generators and the power distribution is detected by using a microwave spectrum analyzer. The frequencies of the sources differ by $1 \mathrm{MHz}$ to ensure that the sources are entirely incoherent. The reason behind using incoherent sources is to prevent the contribution of interference effects to the imaging resolution measurements. ${ }^{13}$ The measured power distribution of sources, separated by $\lambda / 8$, is plotted by the black line ( $)$ in Fig. 4. As seen in the figure, the peaks of two sources are clearly resolved. The resolution becomes better for $\lambda / 5$ separation between the sources (red line, $\nabla$ ). Finally, when the sources are $\lambda / 3$ apart (blue line, $\mathbf{\square}$ ), two peaks are entirely resolved. In order to avoid any possible channeling effects, the sources are intentionally not placed at the line of SRRwire boards. Besides, the distances between the sources in all three experiments are carefully chosen such that they are not multiples of the lattice constant. The lattice constant is on the order of $\lambda / 8.5$; therefore the NIM structure behaves as an effective medium. The periodicity has a significant effect on the resolution of the superlens by limiting the recovery of evanescent components. ${ }^{\text {t7 }}$

Following the analysis by Smith et al. where they discussed the effect of deviation from the ideal parameters on subwavelength resolution, one may argue that superlens with $\varepsilon=\mu=-1.8$ may not provide $\lambda / 8$ resolution. We think that
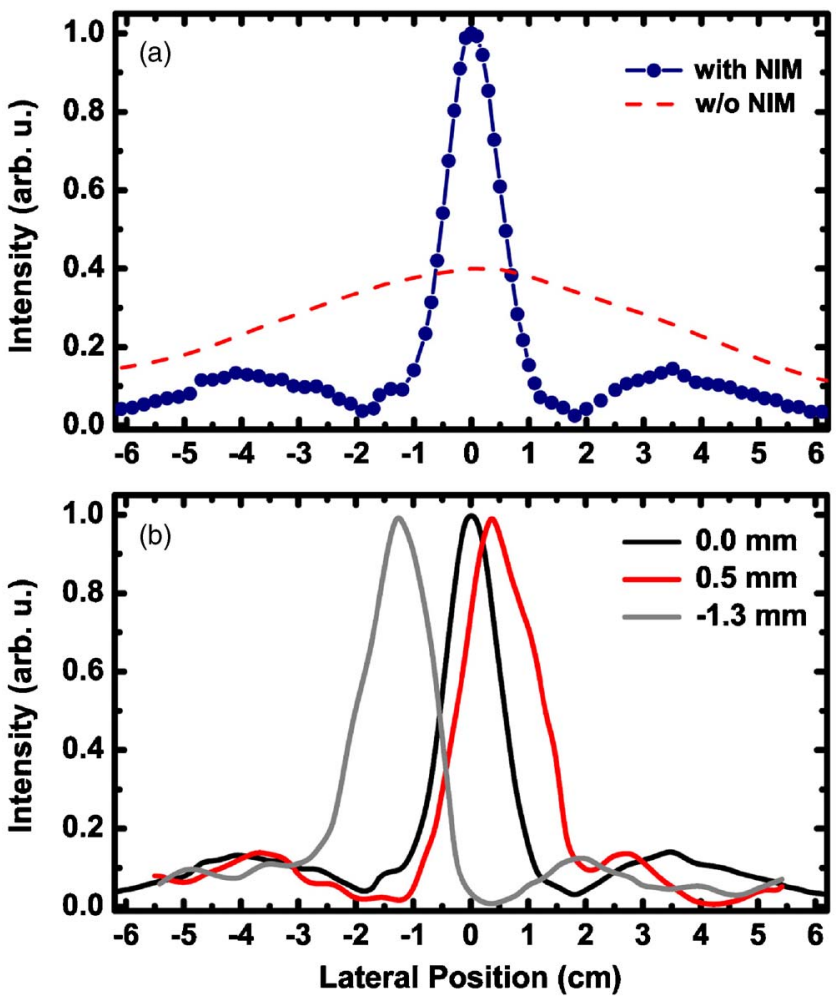

FIG. 3. (Color online) The measured power distributions at the image plane (a) with (blue) and without (dashed red line) NIM superlens. Normalized intensity in free space is multiplied with 0.4 in the figure. (b) The field profiles from single sources placed at three different locations along the $x$ direction that are $x=0 \mathrm{~cm}$ (black line, 0 ), $0.5 \mathrm{~cm}$ (red line, $\nabla$ ) and $-1.3 \mathrm{~cm}$ (gray line, $\mathbf{a}$ ). 


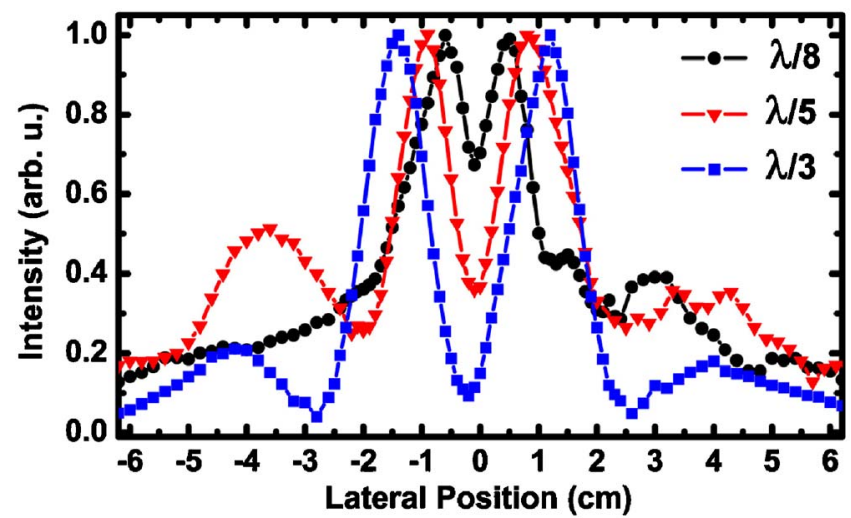

FIG. 4. (Color online) The measured power distributions for two point sources separated with distances of $\lambda / 8$ (black line), $\lambda / 5$ (red line), and $\lambda / 3$ (blue line). The normalized intensity in free space is shown with a green dashed-dotted line and multiplied with 0.2 in the figure.

anisotropic effects take place in our superlens and the observed high resolution may be attributed to inherent anisotropy of our structure. The effect of anisotropy on imaging performance is discussed by Lagarkov et al. ${ }^{7}$

In the near-field regime, the electrostatic and magnetostatic limits apply, and therefore, the electric and magnetic responses of materials can be treated as decoupled. ${ }^{12}$ This in turn brings the possibility of constructing superlenses from materials with negative permittivity, 14 or negative permeability. ${ }^{15}$ Recently, Wiltshire et al. ${ }^{15}$ reported $\lambda / 64$ resolution that is obtained from a magnetostatic superlens operating at radio frequencies with an effective permeability value of $\mu_{\mathrm{eff}}=-1$. The advantage of using negative-index lenses over negative-permittivity or negative-permeability lenses is that the subwavelength resolution can be obtained for both transverse-electric and transverse-magnetic polarizations of EM waves. However, single-negative lenses can only focus EM waves with one particular polarization.

Superlenses can be used in several applications such as imaging, sensing, and subwavelength nanolithography. Here, we verified that it is possible to obtain subdiffraction resolution from a microwave superlens with an effective negative refractive index. Since the NIMs are gearing toward optical frequencies, ${ }^{20-22}$ we believe that subwavelength resolution can be achieved at visible wavelengths by employing thin
NIM superlenses. However, meticulous designs are needed to achieve low loss, impedance-matched superlenses at optical frequencies, since the amount of absorption losses is relatively large compared to the losses at microwave frequencies.

This work is supported by the European Union under the projects EU-NoE-METAMORPHOSE, EU-NoEPHOREMOST, and TUBITAK under Projects Nos. 104E090, 105E066, 105A005, and 106A017. One of the authors (E.O.) also acknowledges partial support from the Turkish Academy of Sciences.

${ }^{1}$ R. A. Shelby, D. R. Smith, and S. Schultz, Science 292, 77 (2001).

${ }^{2}$ E. Cubukcu, K. Aydin, E. Ozbay, S. Foteinopoulou, and C. M. Soukoulis, Nature (London) 423, 604 (2003).

${ }^{3}$ V. G. Veselago, Sov. Phys. Usp. 10, 509 (1968).

${ }^{4}$ D. R. Smith, W. J. Padilla, D. C. Vier, S. C. Nemat-Nasser, and S. Schultz, Phys. Rev. Lett. 84, 4184 (2000).

${ }^{5}$ K. Aydin, K. Guven, L. Zhang, M. Kafesaki, C. M. Soukoulis, and E. Ozbay, Opt. Lett. 29, 2623 (2004).

${ }^{6}$ A. Grbic and G. V. Eleftheriades, Phys. Rev. Lett. 92, 117403 (2004).

${ }^{7}$ A. N. Lagarkov and V. N. Kissel, Phys. Rev. Lett. 92, 077401 (2004).

${ }^{8}$ K. Aydin, I. Bulu, and E. Ozbay, Opt. Express 13, 8753 (2005).

${ }^{9}$ N. Fang, H. Lee, C. Sun, and X. Zhang, Science 308, 534 (2005).

${ }^{10}$ K. Aydin, K. Guven, C. M. Soukoulis, and E. Ozbay, Appl. Phys. Lett. 86, 124102 (2005).

${ }^{11}$ K. Aydin, I. Bulu, K. Guven, M. Kafesaki, C. M. Soukoulis, and E. Ozbay, New J. Phys. 7, 168 (2005).

${ }^{12}$ J. B. Pendry, Phys. Rev. Lett. 85, 3966 (2000).

${ }^{13}$ E. Cubukcu, K. Aydin, S. Foteinopolou, C. M. Soukoulis, and E. Ozbay, Phys. Rev. Lett. 91, 207401 (2003).

${ }^{14}$ R. J. Blaikie and D. O. S. Melville, J. Opt. A, Pure Appl. Opt. 7, S176 (2005).

${ }^{15}$ M. C. K. Wiltshire, J. B. Pendry, and J. V. Hajnal, J. Phys.: Condens. Matter 18, L315 (2006).

${ }^{16}$ N. Fang and X. Zhang, Appl. Phys. Lett. 82, 161 (2003).

${ }^{17}$ D. R. Smith, D. Schurig, M. Rosenbluth, S. Schultz, S. A. Ramakrishna, and J. B. Pendry, Appl. Phys. Lett. 82, 1506 (2003).

${ }^{18}$ V. A. Podolskiy and E. E. Narimanov, Opt. Lett. 30, 75 (2005).

${ }^{19}$ X. Chen, T. M. Grzegorczyk, B.-I. Wu, J. Pacheco, Jr., and J. A. Kong, Phys. Rev. E 70, 016608 (2004).

${ }^{20}$ S. Zhang, W. Fan, N. C. Panoiu, K. J. Malloy, R. M. Osgood, and S. R. J. Brueck, Phys. Rev. Lett. 95, 137404 (2005).

${ }^{21}$ V. M. Shalaev, W. Cai, U. K. Chettiar, H. Yuan, A. K. Sarychev, V. P. Drachev, and V. Kildishev, Opt. Lett. 30, 3356 (2005).

${ }^{22}$ G. Dolling, M. Wegener, C. M. Soukoulis, and S. Linden, Opt. Lett. 32, 53 (2007).

${ }^{23}$ P. V. Parimi, W. T. Lu, P. Vodo, and S. Sridhar, Nature (London) 426, 404 (2003). 